\title{
Advances in cancer immunotherapy 2019 - latest trends
}

\author{
Stephan Kruger ${ }^{1,3^{*}+}$, Matthias IImer ${ }^{2,6 \dagger}{ }^{4}$, Sebastian Kobold ${ }^{3}$, Bruno L. Cadilha ${ }^{3}$, Stefan Endres ${ }^{3}$, Steffen Ormanns ${ }^{4}$, \\ Gesa Schuebbe1, Bernhard W. Renz ${ }^{2,6}$, Jan G. D'Haese ${ }^{2}$, Hans Schloesser ${ }^{5}$, Volker Heinemann ${ }^{1,6}$, \\ Marion Subklewe ${ }^{1,6,8}$, Stefan Boeck ${ }^{1,6}$, Jens Werner ${ }^{2}$ and Michael von Bergwelt-Baildon ${ }^{1,6,7,8}$
}

\begin{abstract}
Immunotherapy has become an established pillar of cancer treatment improving the prognosis of many patients with a broad variety of hematological and solid malignancies. The two main drivers behind this success are checkpoint inhibitors (CPIs) and chimeric antigen receptor (CAR) T cells. This review summarizes seminal findings from clinical and translational studies recently presented or published at important meetings or in top-tier journals, respectively. For checkpoint blockade, current studies focus on combinational approaches, perioperative use, new tumor entities, response prediction, toxicity management and use in special patient populations. Regarding cellular immunotherapy, recent studies confirmed safety and efficacy of CAR T cells in larger cohorts of patients with acute lymphoblastic leukemia or diffuse large B cell lymphoma. Different strategies to translate the striking success of CAR T cells in B cell malignancies to other hematological and solid cancer types are currently under clinical investigation. Regarding the regional distribution of registered clinical immunotherapy trials a shift from PD-1 / PD-L1 trials (mainly performed in the US and Europe) to CAR T cell trials (majority of trials performed in the US and China) can be noted.
\end{abstract}

Keywords: Immunotherapy, Programmed cell death protein 1 (PD-1), Programmed cell death protein ligand 1 (PD-L1), Chimeric antigen receptor T cells (CAR T cells), Trends, Regional distribution

\section{Background}

The importance of immunotherapy has been acknowledged by the Nobel prize for physiology or medicine 2018 awarded for the discovery of cytotoxic T-lymphocyteassociated protein (CTLA-4) to James P. Allison and programmed cell death protein 1 / programmed cell death protein ligand 1 (PD-1 / PD-L1) to Tasuku Honjo [1]. Malignant tumors take advantage of the inhibitory PD-1 / PD-L1 or CTLA-4 pathways to evade the immune system [2]. Disruption of this axis by blocking monoclonal antibodies can induce durable remissions in different cancer types and has led to numerous FDA and EMA approvals, among others, for the treatment of melanoma, lung cancer, urothelial cancer, head and neck

\footnotetext{
*Correspondence: stephan.kruger@med.uni-muenchen.de

${ }^{\dagger}$ Stephan Kruger and Matthias IImer contributed equally to this work.

'Department of Medicine III, University Hospital Munich, LMU Munich, Marchioninistr. 15, D-81377 Munich, Germany

${ }^{3}$ Center of Integrated Protein Science Munich (CIPS-M) and Division of Clinical Pharmacology, Department of Medicine IV, University Hospital, LMU Munich, Munich, Germany

Full list of author information is available at the end of the article
}

squamous cell carcinoma (HNSCC), renal cell cancer (RCC) and Hodgkin's disease [3]. Up-to-date reviews providing a comprehensive overview of approved indications for different CPIs have been published previously [3, 4].

This review focuses on clinical and pre-clinical findings that might guide future clinical application of CPIs in general. We identified potentially trendsetting studies on CPIs for combinational approaches, perioperative use, new tumor entities, response prediction, toxicity management and use in special patient populations. Further, we identified studies focusing on efficacy and toxicity of anti- CD19 CAR T cells in larger patient cohorts as well as seminal findings on adoptive $\mathrm{T}$ cell therapy in other hematological and solid malignancies.

\section{Checkpoint inhibitors \\ Combinational therapy Combination with chemotherapy}

Traditionally, chemotherapy and radiotherapy were believed to mediate their anti-cancer effect by direct killing 
of cancer cells. This concept was challenged over a decade ago by Zitvogel and co-workers who discovered that the antineoplastic effect of chemotherapy, in part, depends on the immunogenic cell death of cancer cells. This leads to immune stimulatory signals via activation of the innate immune system through pattern recognition receptors such as toll-like receptor 4 (TLR4) [5]. Different studies confirmed the immunological effects of chemotherapeutic drugs, in particular, platinum-based agents, and paved the way for the development of combinational regimens using PD-1 / PD-L1-blockade together with established chemotherapeutic drugs [6-11]. Last year saw the completion of several practicechanging phase III trials showing the efficacy of combining PD-1 / PD-L1-blockade with chemotherapy in small cell lung cancer (SCLC), non-small cell lung cancer (NSCLC), HNSCC and breast cancer [12-15]. Currently, more than 170 studies are investigating the promising combination of PD-1/PD-L1 blockade plus chemotherapy in different cancer entities [4].

\section{Combination with radiotherapy}

Anecdotal reports on systemic anti-tumor response after irradiation of a single tumor lesion date back more than one century [16]. Regression of non-irradiated lesions after localized radiotherapy of a single lesion was first termed 'abscopal effect' in 1958 [17]. The underlying mechanism remained unexplained for a long period and it took almost another 50 years, before Demaria et al. concluded that "Ionizing radiation inhibition of distant untreated tumors (abscopal effect) is immune mediated" [18]. Nowadays, the causative link between local radiation, immunogenic cell death and systemic tumor response is well-established [19]. While the abscopal effect remains a sporadic event, numerous strategies are now under investigation to harness the immunogenic effect of radiotherapy [19].

Given the clinical success of checkpoint blockade, combining radiotherapy with PD-1 / PD-L1 blockade is of special interest. Pre-clinical evidence highlights the synergistic potential of this combination [20]. Translational results from an ongoing phase I/II trial (NCT01976585) investigating local radiotherapy in combination with local application of immunostimulatory agents in patients with indolent lymphoma further support the combination of radiotherapy and PD-1 / PD-L1 blockade [21]. In this trial, patients received 2 Gy of local radiotherapy as part of a socalled "in situ vaccination" (ISV: radiotherapy plus intratumoral application of Fms-related tyrosine kinase 3 ligand [Flt3L] and a Toll-like receptor 3 [TLR3] ligand). ISV was able to induce systemic ("abscopal") tumor regression in three out of eleven treated patients. Importantly, in nonresponding patients, the induction of tumor infiltrating PD$1^{+} \mathrm{CD}^{+} \mathrm{T}$ cells was observed, prompting a follow-up trial, which is now recruiting patients for ISV in combination with PD-1 blockade (NCT03789097).

Despite these encouraging findings, negative results for the combination of radiotherapy and checkpointblockade have also been recently reported. In a phase II trial in metastatic HNSCC, the addition of local radiotherapy to systemic PD-1 blockade was not able to boost the effect of PD-1-blockade. Here, patients were randomized to receive either nivolumab monotherapy or nivolumab plus stereotactic body radiation therapy (SBRT) of a single tumor lesion. The primary study endpoint - response rate in none-irradiated tumor lesions was not met. Response rate in patients receiving nivolumab plus SBRT was $22.2 \%$ (95\% confidence interval $[\mathrm{CI}]$ : 10.6-40.8\%) versus $26.9 \%$ (95\% CI: $13.7-46.1 \%$ ) for single agent nivolumab [22].

The placebo-controlled, randomized phase III PACIFIC trial investigated the addition of durvalumab (anti-PD-L1) to platinum-based chemoradiotherapy in locally advanced (stage III) NSCLC. The addition of durvalumab resulted in an impressive increase in progression-free (PFS) and overall survival (OS) (17.2 versus 5.2 (PFS) and 28.7 months versus "not reached" (OS), respectively) [23, 24]. In this context, the timely administration of PD-1 blockade appeared to be important: patients receiving durvalumab within 14 days after completion of chemoradiotherapy had a better overall survival than patients starting durvalumab-treatment at a later time point [25].

While recent results encourage further in-depth investigation of checkpoint blockade plus radiotherapy, successful concepts might depend on additional combination partners like the above-mentioned in situ-vaccination or chemotherapy. Additional well-designed clinical trials are necessary to identify optimal strategies for combinations and treatment sequences.

\section{Combination with immunomodulatory drugs}

The first CPI approved for clinical use was ipilimumab, targeting CTLA-4. Given the success of ipilimumab and the even greater success of PD-1-blockade, it is not surprising, that - with more than 250 clinical trials - the combination of PD-1 and CTLA-4 blockade is the most vigorously investigated combinational approach of two immunomodulatory drugs [4].

Due to the large number of clinically approved immunomodulatory agents (currently more than 25 ) and many more in pre-clinical and clinical development, there is an almost infinite number of combinatorial regimens for further clinical evaluation. In this regard, it is important to note, that the combination of two immunomodulatory drugs can also have antagonistic instead of synergistic effects [26]. Wise selection strategies based on preclinical data to select combinatorial approaches for 
clinical testing are important [26]. In light of this, Tauriello et al. provided an example for an elaborate preclinical model system. By using a quadruple mutant colorectal mouse model, they were able to recapitulate important immunological hallmarks of microsatellite stable colorectal cancer (MSS CRC) [27]. While PD-1 / PD-L1 blockade showed only marginal efficacy in this setting paralleling results of clinical trials with PD-1/PD-L1 blockade in MSS CRC, impressive effects were achieved when PD-1/PD-L1 blockade was combined with inhibition of transforming growth factor beta (TGF- $\beta$ ) [27].

Building on pre-clinical and early clinical data for simultaneous targeting of CD40 and PD-1 / PD-L1 in pancreatic cancer (a disease for which all immunotherapeutic efforts have failed so far), a phase I trial investigating the combination of CD40, durvalumab and chemotherapy was initiated. The promising results were recently presented at the annual meeting of the AACR (2019), making this combinational strategy one to keep track of in the years to come [28-30].

\section{Peri-operative use}

Up to now, the clinical use of CPIs has been mainly restricted to advanced tumor stages. Yet, efficacy of checkpoint blockade has been reported to be dependent on baseline tumor burden (with better efficacy observed in patients with low tumor burden), making peri-operative usage of checkpoint blockade an attractive treatment option from a theoretical point of view [31, 32].

Although ipilimumab was approved for the adjuvant treatment of melanoma patients by the FDA (but not by the EMA) based on a placebo-controlled phase III trial reporting superior recurrence-free and overall rates, its use was internationally disputed given the relatively high frequency of serious immune-related adverse events in patients receiving treatment with ipilimumab [33-35]. In Europe, nivolumab was the first checkpoint inhibitor approved for adjuvant treatment of melanoma patients, based on results of the CheckMate 238 study reported in 2017 [36]. In this study, nivolumab was compared to ipilimumab as adjuvant therapy for patients after resection of stage III-IV melanoma. Recurrence-free survival was reported to be superior while severe adverse events were significantly lower in patients treated with nivolumab (12-month recurrence-free survival: $70.5 \%$ vs $60.5 \%$; grade 3 or 4 adverse events: $14.4 \%$ versus $45.9 \%$ for patients receiving nivolumab or ipilimumab, respectively).

A logical next step to consider would be neoadjuvant use of CPIs. Theoretically, neoadjuvant immunotherapy might be able to prime systemic immunity for tumor surveillance after complete resection - at a time point when tumor antigens are still abundantly present [37]. This concept is supported by recent translational findings from an early clinical study in patients with resectable melanoma: in a randomized phase Ib study, neoadjuvant treatment with nivolumab and ipilimumab induced a higher number of tumor specific $\mathrm{T}$ cell clones than adjuvant treatment [38]. Early clinical findings reported from patients with NSCLC, HNSCC and microsatellite unstable (MSI) CRC further emphasize the high potential of neoadjuvant treatment [39-41]. In the latter study, seven out of seven patients with MSI CRC (100\%) responded to neoadjuvant treatment with complete remissions observed in 4/7 (57\%) patients [41].

A large number of clinical trials is currently investigating neoadjuvant immunotherapy for different disease entities (for example, we identified nine clinical trials for neoadjuvant anti- PD-1 / PD-L1 treatment in NSCLC: NCT03197467, NCT02938624, NCT02259621, NCT03 694236, NCT03732664, NCT02994576, NCT03030131, NCT02716038, NCT02818920). Given the considerable side effects of checkpoint blockade - particularly, if administered as combinational therapy - wise selection of patients that might benefit from neoadjuvant or adjuvant treatment is mandatory. One possibility for adjuvant treatment stratification might be detection of minimal residual disease (MRD) by circulating tumor DNA (ctDNA), a strategy, that is currently investigated by a clinical trial in triple-negative breast cancer (TNBC) (NCT03145961) [42].

\section{New tumor entities}

Current studies show the efficacy of CPIs in patients with malignant melanoma (MM), NSCLC or neoplasms with mutational defects in DNA mismatch repair proteins (micro satellite instability or MSI) independent of the actual tumor entity. Intriguingly, all of these tumors share a relatively high mutational load when their genetic characteristics are comparatively analyzed [43]. This common characteristic leads to increased expression of neo antigens in the tumor, stimulating an increased infiltration of the tumor by immune cells, which in turn can be "activated" by CPI administration. This fact can also be used to explain why CPI studies in certain tumor entities (among others pancreatic ductal adenocarcinoma (PDAC) or colorectal carcinoma (CRC) without DNA mismatch repair protein defects) haven't been successful as of yet.

On average, breast cancer and AML are also characterized by a low mutational load [43]. With that background, two remarkable studies from 2018 should be mentioned here in more detail. On the one hand, the phase III trial IMpassion130 tested the combination of atezolizumab (anti-PD-L1) plus nab-paclitaxel versus nab-paclitaxel monotherapy in treatment-naïve patients with metastatic, triple-negative breast cancer (TNBC). The addition of atezolizumab not only improved the patients' PFS (PFS), but also their overall survival (OS) 
[14]. For patients with TNBC, this was the first phase III study that showed a strong benefit of targeted (immune) therapy. A total of 144 studies on PD-1 / PD-L1 blockade in TNBC are currently registered on clinicaltrials.gov (Fig. 1a).

On the other hand, for AML, data on nivolumab maintenance therapy in high-risk AML patients was presented at the annual meeting of the American Society of Clinical Oncology (ASCO) in 2018. This study investigated whether the administration of nivolumab might prolong the time of complete remission (CR) in patients that do not qualify for an allogenic stem cell transplantation. In 14 patients that were followed-up for a median of 19.3 months, the median duration of CR averaged 8.3 months, whereas the median OS had not been reached at the time of presentation of the data. Despite the very limited number of patients, this study shows an exciting treatment concept for this specific treatment group [44].

In conclusion, both studies exemplify that successful CPI concepts might also be feasible for tumor entities with a low mutational load. Numerous clinical trials are currently investigating the use of CPIs in different cancer entities (Fig. 1a). It will be interesting to see whether further positive results for tumor entities with low mutational burden will follow in the future.

\section{Biomarkers for response prediction of checkpoint blockade}

Determination of PD-L1 expression by immunohistochemistry is an FDA-approved diagnostic test and a prerequisite for treatment with anti-PD-1 / PD-L1 therapy in various indications (e.g. monotherapy treatment of urothelial cancer with atezolizumab or pembrolizumab). However, determining PD-L1 expression does not identify all patients that profit from anti-PD-1 / PD-L1 therapy, highlighting the need for additional and better biomarkers [45].

\section{Tissue biomarkers}

Microsatellite instability and tumor mutational burden Another approved biomarker test (for pembrolizumab) is the determination of microsatellite instability (MSI) or deficient mismatch repair (dMMR). Pembrolizumab was the first drug that was FDA-approved with a "tumor-agnostic" indication based on findings from five different clinical trials including 15 tumor entities with MSI/dMMR tumors (KEYNOTE -012, - 016, - 028, 158 and - 164). MSI/dMMR results in increased tumor mutational burden (TMB) with subsequent increase in neoantigens and immune cell infiltration, rendering tumors susceptible to PD-1 /PD-L1 blockade [46]. In different studies, the direct determination of TMB was also established as predictive biomarker for immunotherapy [47-49]. However, recently presented data suggests that not all patients with MSI/dMMR tumors also have a high TMB [50]. Furthermore, $\mathrm{TMB}^{\text {high }}$ is also observed in the absence of MSI/dMMR [46]. More studies are therefore necessary to inform strategies on selection of MSI/dMMR or TMB as biomarker for response to checkpoint blockade.

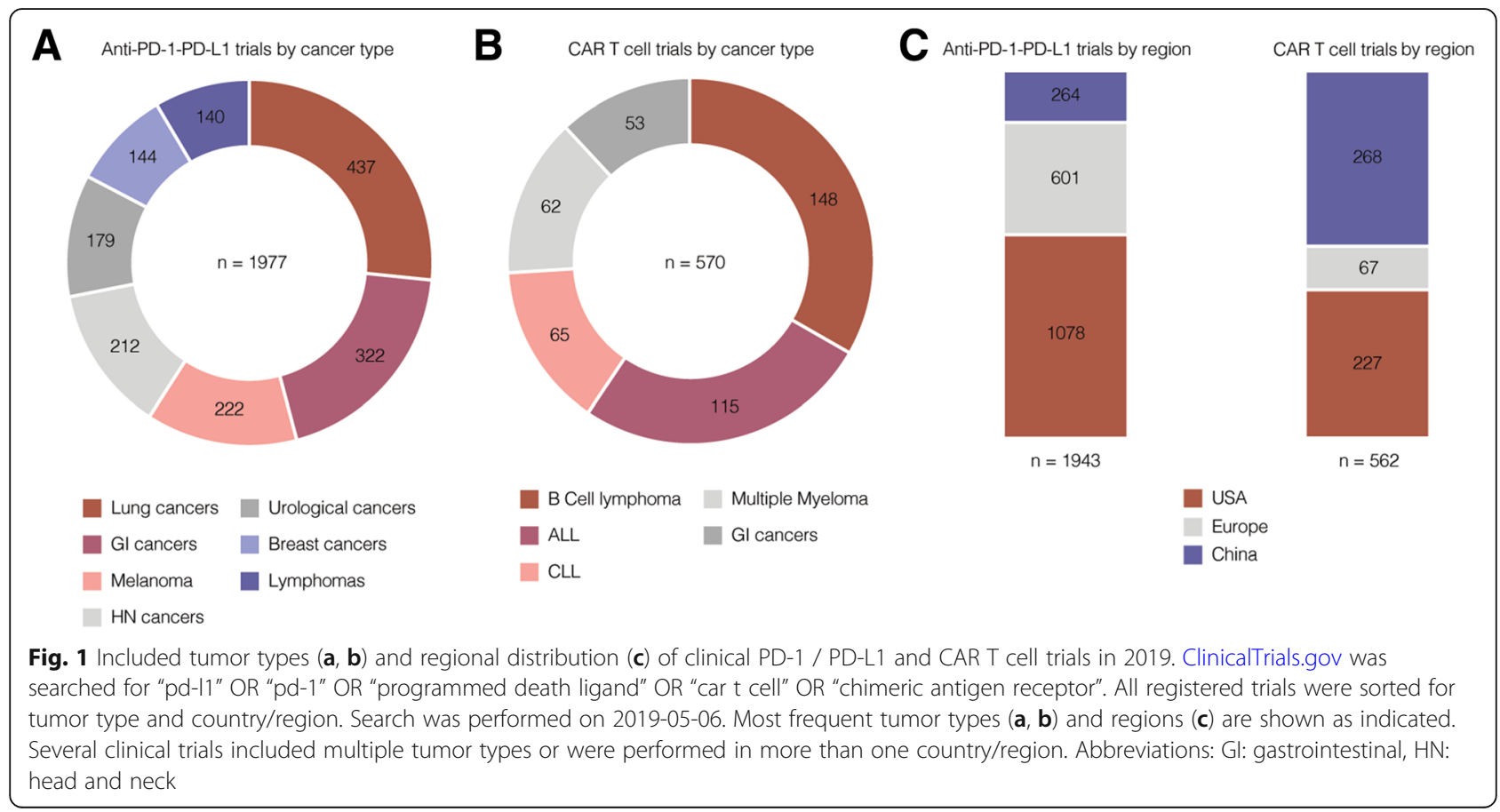


Tumor mutational burden and PD-L1 expression It was previously described that TMB does not correlate to PD-L1 expression [51]. This finding was confirmed and put into therapeutic context by the ChekMate227 trial [52]. In this trial, NSCLC patients were stratified according to tumoral PD-L1 expression ( $\geq 1 \%$ vs $<1 \%$ ). Patients were then randomized (1:1:1) between either chemotherapy, nivolumab (nivolumab plus chemotherapy for patients with $<1 \%$ PD-L1 expression, respectively) or nivolumab plus ipilimumab. One predefined endpoint was response rate in patients with a $\mathrm{TMB}^{\text {high }}$ (defined as $>10$ mutations per megabase). Independent of PD-L1 expression, nivolumab plus ipilimumab was superior to chemotherapy in patients with high TMB [52].

Inflammatory gene signatures Apart from the biomarkers mentioned above, different inflammatory TMBsignatures determined in tumor tissues can serve as biomarkers for checkpoint blockade. These signatures indicate infiltration by a specific immune cell subset (e.g. effector T cells) or activation of a specific signaling pathway (e.g. interferon- $\gamma$ signaling). Recently published data from the IMmotion150 trial suggests that these signatures could even be superior to TMB in patients with metastatic renal cell carcinoma: patients were randomized between the combination of atezolizumab (anti-PDL1) +/- bevacizumab versus sunitinib. T-effector, interferon- $\gamma$ and myeloid inflammatory gene expression signatures were superior to TMB in predicting response to atezolizumab [53]. It should be noted, that these analyses were exploratory.

Further research is necessary to integrate the aforementioned tissue biomarkers into one clinical applicable diagnostic algorithm. Well-designed translational studies might also be able to identify completely new tissue biomarkers to predict clinical response to CPI treatment. One example are gene fusions producing immunogenic neoantigens. Such gene fusions were recently shown to predict response to checkpoint blockade in HNSCC patients with low TMB and minimal immune cell infiltrate [54].

\section{Soluble biomarkers}

Identifying soluble biomarkers for response prediction in peripheral blood would have several advantages over tissue biomarkers. For instance, they are easily and noninvasively accessible and can be sampled repetitively for continuous response prediction. The soluble forms of PD-1 and PD-L1 (sPD1 and sPD-L1) are also present in the peripheral blood $[55,56]$. Only few studies have investigated SPD-1 and SPDL-1 as biomarkers for response to checkpoint blockade. One small study in NSCLC patients suggested that high SPD-L1 levels predict poor response to nivolumab [57], a finding that is somewhat contrary to tissue PD-L1, because high PD-L1 tissue expression indicates higher likelihood of response to checkpoint blockade. Findings from patients with pancreatic cancer suggest that SPD-1 and SPD-L1 are rather indicators of systemic inflammation and independent from tumoral PD-L1 expression [56]. Together these findings question the aptitude of sPD-1 and sPD-L1 as biomarkers for checkpoint blockade.

An emerging soluble biomarker for checkpoint blockade is ctDNA in peripheral blood. It can be used for different applications. First, ctDNA can be used to determine tumor mutational burden (TMB) [58]. TMB measured in peripheral blood has been shown to predict response to checkpoint blockade in NSCLC patients [58, 59]. In patients receiving conventional chemotherapy, repeated ctDNA measurement can be used for early response prediction [60]. Recently published studies suggest that changes in ctDNA levels can also be early predictors for response to immunotherapy [61, 62]. Importantly, it might also aid to distinguish pseudoprogression from truly progressive disease in patients treated with immunotherapy [63].

\section{Immune related adverse events as biomarker for tumor response}

Different studies suggested that immune related adverse events (IrAEs) indicate response to checkpoint blockade $[64,65]$. These studies, however, were not controlled for lead time bias [66] and it is therefore not clear, whether IrAEs are truly independent predictors for response or merely reflect a longer time under treatment. Recent studies controlled for lead-time bias reported conflicting data: a large monocentric study including different cancer types presented at ESMO 2018 did not find a correlation between IrAEs and response to checkpoint blockade after controlling for lead-time bias [67]. Yet, another recent study in renal cell carcinoma reported better efficacy of nivolumab in patients with IrAEs after controlling for lead-time bias [68].

\section{Toxicity management Use of steroids}

The occurrence of immune-mediated side effects (e.g. colitis, autoimmune hepatitis, endocrine or neurological side effects) requires treatment with glucocorticoids (e.g. prednisolone) as early as possible depending on the severity [69]. Whether the use of glucocorticoids has a negative effect on the success of CPI treatment remains controversial. A study presented at the annual meeting of the ASCO in 2018 retrospectively investigated NSCLC patients who received glucocorticoids at the beginning of CPI therapy. The reasons for glucocorticoid administration included the treatment of symptoms caused by brain metastases as well as respiratory distress or fatigue. In a multivariate analysis which included 
performance status and presence of brain metastases, patients who received glucocorticoids at the start of treatment responded significantly worse to CPI administration [67]. On the other hand, as mentioned in the biomarker section, it is often postulated that patients who develop immune-mediated side effects (and receive glucocorticoids) benefit from CPI therapy over a longer period of time (or at least not shorter) than patients without immune-mediated side effects.

As a practice-based approach, immune-mediated side effects (depending on the severity and type of side effects) should be treated early with glucocorticoids to prevent permanent damage [69]. On the other hand, the need for symptomatic and sustained administration of steroids for other reasons (e.g. brain metastases or respiratory distress) during CPI therapy should be critically scrutinized in everyday clinical practice.

\section{Special populations: patients with pre-existing autoimmune disease or HIV}

Most clinical trials on CPI therapy have excluded patients with pre-existing autoimmune diseases or human immunodeficiency virus infection (HIV). In this regard, it remained unclear whether a CPI therapy is also conceivable in these patients.

The safety and efficacy of CPIs in patients with preexisting autoimmune diseases has been recently studied in a French registry study including different tumor entities [70]. Patients with and without pre-existing autoimmune diseases were included (patients with preexisting autoimmune disease: $n=45$, patients without pre-existing autoimmune disease: $n=352$ ). Although the incidence of immune-mediated side effects was significantly increased in the group of patients with preexisting autoimmune diseases (44\% versus $23 \%$ ), there was no difference in overall survival between the two groups.

For the use of CPIs in patients with HIV, data from a small HIV-positive cohort of patients $(n=20)$ with NSCLC or multiple myeloma was presented at the annual meeting of the European Society of Medical Oncology (ESMO) in 2018. Overall, the therapy with CPIs was well tolerated in patients with HIV and no immunemediated side effects were observed. An increase in HIV viral load was observed only in one patient who had paused his antiretroviral therapy. A response to therapy (PR or CR) was observed in $24 \%$ of patients [71].

Overall, both studies suggest that CPI therapy might be feasible and effective in patients with pre-existing autoimmune disease or HIV. Due to limited data on these special patient groups, a careful assessment of potential benefit versus potential harm is mandatory before starting CPI therapy in these patients.

\section{Cellular immunotherapy}

Chimeric antigen receptor T cells

Tisagenlecleucel and axicabtagen-ciloleucel were the first two cellular cancer immunotherapies receiving FDA and EMA approval in 2017 and 2018, respectively. They are approved to treat patients with acute lymphoblastic leukemia (ALL, tisagenlecleucel) and diffuse-large B cell lymphoma (DLBCL, tisagenlecleucel and axicabtagenciloleucel). Approval was based on impressive response rates observed in the ELIANA trial (relapsed or refractory $[\mathrm{r} / \mathrm{r}]$ ALL in pediatric patients or young adults treated with tisagenlecleucel), JULIETH trial ( $\mathrm{r} / \mathrm{r}$ DLBCL, tisagenlecleucel) and ZUMA-1 trial ( $\mathrm{r} / \mathrm{r}$ DLBCL, axicabtagen-ciloleucel) [72-74].

Tisagenlecleucel and axicabtagen-ciloleucel are autologous T cell products. After leukapheresis, T cells are genetically engineered to express an anti-CD19 chimeric antigen receptor (anti-CD19 CAR T cells). Re-infusion of CAR $\mathrm{T}$ cells is preceded by a lympho-depleting chemotherapy to allow for subsequent in vivo expansion of CAR T cells (Fig. 2).

Numerous clinical trials (as of May 2019 more than 550, Fig. 1b) are investigating CAR $T$ cell therapies for different hematological and solid cancer types [75]. Of interest and in harsh contrast to trials on PD-1 / PD-L1 blockade is the regional distribution of clinical trials on CAR T cell therapy (Fig. 1c). The USA and China by far outcompete the EU in terms of registered CAR T cell trials. This regional imbalance has been described and discussed previously and should be addressed by researches and health care policy makers in the European Union [76].

Recently reported studies on cellular therapy mainly addressed two important questions: (I) Long term and "real world" experience regarding toxicity and efficacy of CAR T cells (II) Can the striking success of CAR T cells in ALL and DLBCL be translated to other hematological and - more importantly - solid malignancies?

\section{Updated results from CD19 CAR T cells clinical trials}

Follow-up results for efficacy and toxicity from the ELIANA, JULIETH and ZUMA-1 trial were recently presented at the annual meetings of the European Hematology Association (EHA) and the American Society of Hematology (ASH).

Efficacy As of 2018, 97 patients aged $\leq 21$ years with $\mathrm{r} / \mathrm{r}$ ALL were enrolled in the ELIANA trial, 79 patients were infused with CD19 CAR T cells and a complete remission was achieved in 65 patients. After a median followup of 24 months, response was ongoing in 29 patients (45\%), with a maximum (ongoing) duration of response of 29 months [77]. For $\mathrm{r} / \mathrm{r}$ DLBCL patients treated with tisagenlecleucel, the updated analysis presented at EHA 2018 included 111 infused patients. Overall response 


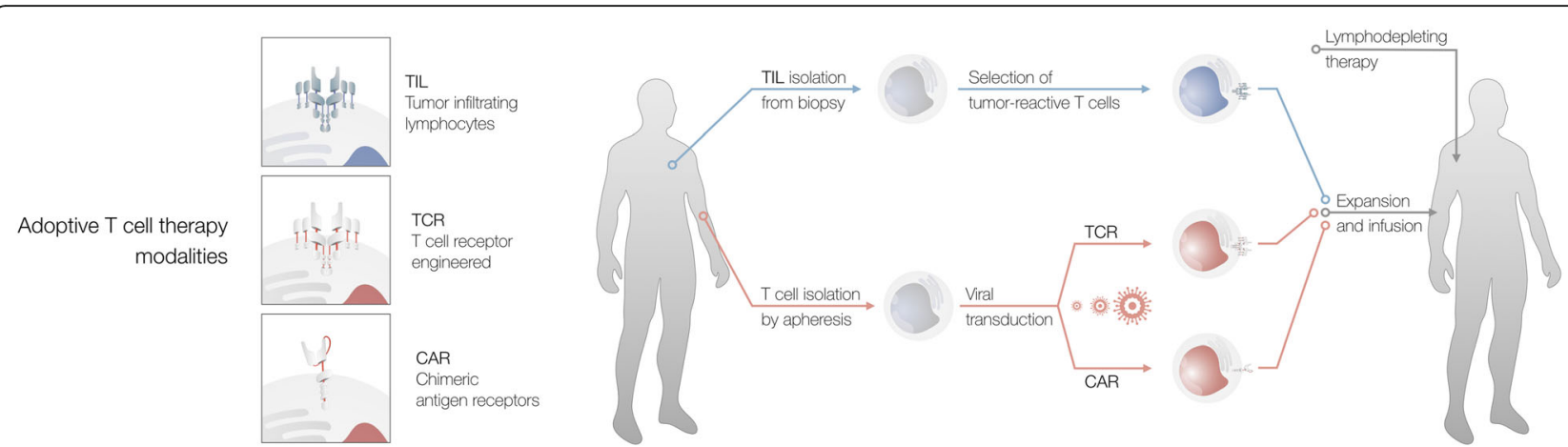

Fig. 2 Different strategies for adoptive T cell therapy. Abbreviations: CAR: chimeric antigen receptor, TCR: T cell receptor, TIL: tumor infiltrating lymphocytes

rate (ORR) was $52 \%(40 \% \mathrm{CR}, 12 \% \mathrm{PR})$ [78]. After a median follow-up time of 14 months, median duration of response was not reached. Median overall survival for all infused patients was 11.7 months [79]. For axicabtagenciloleucel, the 2-year follow-up data was presented at ASH 2018. A total of $108 \mathrm{r} / \mathrm{r}$ DLBCL patients had at least one year of follow-up. ORR in this cohort was $82 \%$ (58\% CR). An ongoing response was observed in $42 \%$ of all patients after a median follow-up of 15.4 months, no updated overall survival data was reported [80].

For axicabtagen-ciloleucel, "real world" efficacy was confirmed by data from seventeen US academic centers who evaluated axicabtagen-ciloleucel outside of clinical trials, independent of the manufacturer after commercialization. The authors reported an ORR of $79 \%(50 \% \mathrm{CR})$, confirming the results reported in the clinical trials mentioned above [81].

While these results support the high therapeutic potential of CAR T cell therapy, a cohort of patients does not respond to - or relapses after - CAR T cell therapy. Considering the latter group (relapse after an initial complete response), it is important to explore further treatment options for these patients. One possibility might be allogeneic stem cell transplantation, which has recently been reported to improve prognosis after antiCD19 CAR T cell therapy for ALL patients who had not received a previous stem cell transplantation [82].

Toxicity The updated data for ELIANA, JULIETH and ZUMA-1 confirm the previously described safety profile with cytokine release syndrome (CRS, incidence of CRS grade $\geq 3: 7$ to $48 \%$ ) and neurologic events (NE, incidence of NE grade $\geq 3: 11$ to $31 \%$ ) as most significant adverse events [78-81].

In the pivotal trials for anti-CD19 CAR $\mathrm{T}$ cells, treatment-related deaths have been reported [77]. No treatment-related deaths were observed in a US multicenter cohort of 165 patients who received axicabtagenciloleucel for $\mathrm{r} / \mathrm{r}$ DLBCL after commercialization outside of clinical trials [81]. Recently, safety of axicabtagenciloleucel was also confirmed in patients $\geq 65$ years [83]. Further it has been reported that neurotoxicity is fully reversible in most patients [84].

While the mentioned results are reassuring regarding saftey of CAR T cell therapy, different strategies are currently under investigation to further improve the safety profile of CAR T cells. These strategies include: (I) modification of the chimeric antigen receptor cell itself [85, 86]; (II) identification of predictive biomarkers for CAR $\mathrm{T}$ cell toxicity [84]; (III) "safety switches" such as inducible suicide genes [87]; and (IV) novel drugs to mitigate CRS and NE [88].

\section{Adoptive $T$ cell therapy in other hematological and solid malignancies}

Chimeric antigen receptor $\mathrm{T}$ cells for hematological and solid malignancies The success of CAR $T$ cells in ALL and B cell lymphoma led to the initiation of numerous follow-up trials in these disease entities (Fig. 1b). Regarding other cancer types, chronic lymphocytic leukemia, multiple myeloma and gastrointestinal cancers are the ones with most clinical CAR T cell trials underway (Fig. 1b).

Additionally, a large variety of strategies to improve efficacy of CAR $\mathrm{T}$ cells in solid malignancies are under pre-clinical investigation [89-94]. Yet, the direct translation of the CAR T cell approach to solid malignancies is often impeded by the lack of a suitable cancer specific antigen resulting in either disappointing efficacy or substantial off target toxicity in early clinical trials [95]. Another important consideration is the tumor environment which is substantially different to the one seen in the above referenced hematological cancers and impedes CAR T cell efficacy [96].

Alternative approaches are genetic modification of the $\mathrm{T}$ cell receptor (TCR) itself or the adoptive transfer of "naturally" occurring tumor reactive $\mathrm{T}$ cells (also termed 
tumor infiltrating lymphocytes or TILs) isolated from autologous tumor tissue or tumor draining lymph nodes (Fig. 2). The manufacturing of TCR-modified T cells is complex, dependent on a specific human leukocyte antigen (HLA)-haplotype and can lead to unexpected offtarget toxicity $[97,98]$. On the other hand, the use of tumor reactive (TCR-native) $\mathrm{T}$ cells has been investigated in numerous clinical studies (mainly in melanoma patients) with promising results $[99,100]$. Recent studies suggest that this approach could also be successfully translated to other solid malignancies.

Ex vivo expansion and reinfusion of autologous tumor reactive $T$ cells $\mathrm{In}$ contrast to CAR $\mathrm{T}$ cells, tumor reactive $\mathrm{T}$ cells recognize tumor cells via their native (unmodified) $\mathrm{T}$ cell receptor (Fig. 2). Tumor reactive $\mathrm{T}$ cells can be isolated from tumor tissue or tumor draining lymph nodes [101-106]. After a potential selection step followed by ex vivo expansion, tumor reactive $\mathrm{T}$ cells are re-infused after lymphodepleting chemotherapy - typically with parallel intravenous administration of interleukin 2 [101]. The high potential of this approach was recently confirmed in melanoma patients after failure of PD-1 / PD-L1 blockade [107] and is currently investigated in a phase III trial as first-line treatment for advanced melanoma patients (NCT02278887). In other solid tumor entities an ongoing early clinical trial (NCT01174121) is currently investigating immunotherapy with tumor reactive $\mathrm{T}$ cells in patients with metastatic gastrointestinal, urothelial, breast, ovarian or endometrial cancer. Case reports from three individual patients described striking responses for this treatment approach for cholangiocarcinoma, colorectal cancer and breast cancer, respectively [104-106]. Further studies are necessary to evaluate the expansion of this promising treatment approach to larger patient populations.

\section{Conclusion}

Immunotherapy of cancer is a rapidly evolving field. Results of currently ongoing studies on checkpoint blockade will most likely expand the use of CPIs to additional patient populations (e.g. new tumor entities, perioperative use, use in special patient populations) and might identify new combination partners for CPI.

The major challenge for adoptive $\mathrm{T}$ cell therapy in years to come is the translation of this treatment modality to solid malignancies. A successful strategy has yet to be defined and might include more advanced genetic engineering of CAR T cells as well as the development of more advanced protocols for the use of tumor reactive (TCR-native) T cells.

Regarding the regional distribution of clinical trials on immunotherapy a shift from the European region (for PD-1 / PD-L1-trials) towards China (leading in terms of number of available CAR $\mathrm{T}$ cell trials) is evident and should be met by intensified research efforts on cellular immunotherapy in Europe.

\begin{abstract}
Abbreviations
ALL: Acute lymphoblastic leukemia; ASCO: American Society of Clinical Oncology; CAR T cells: Chimeric antigen receptor T cells; CPIs: Checkpoint inhibitors; CR: Complete remission; CRC: Colorectal cancer; CRS: Cytokine release syndrome; ctDNA: Circulating tumor DNA; CTLA-4: Cytotoxic Tlymphocyte-associated protein-4; DLBCL: Diffuse-large B cell lymphoma; dMMR: Deficient mismatch repair; EHA: European Hematology Association; EMA: European medicines agency; ESMO: European Society of Medical Oncology; FDA: U. S. food and drug administration; GMP: Good manufacturing practice; HIV: Human immunodeficiency virus; HNSCC: Head and neck squamous cell carcinoma; IrAEs: Immune related adverse events; MSI: Microsatellite unstable; MSS: Microsatellite stable; NE: Neurologic events; NSCLC: Non-small cell lung cancer; ORR: Overall response rate; OS: Overall survival; PD-1: Programmed cell death protein 1; PDAC: Pancreatic ductal adenocarcinoma; PD-L1: Programmed cell death protein ligand 1;

PFS: Progression free survival; PR: Partial remission; r/r: Relapsed or refractory; RCC: Renal cell cancer; RNA: Ribonucleic acid; SBRT: Stereotactic body radiation therapy; SCLC: Small cell lung cancer; SPD1: Soluble form of PD-1; SPD-L1: Soluble form of PD-L1; TCR: T cell receptor; TLR4: Toll-like receptor 4; TMB: Tumor mutational burden
\end{abstract}

\section{Publisher's Note}

Springer Nature remains neutral with regard to jurisdictional claims in published maps and institutional affiliations.

\section{Acknowledgements}

Not applicable

\section{Authors' contributions}

Writing of the manuscript: SK and MI. Conception of the review: SK, MI, SebK, SB and MvB. Figure 1: SK. Figure 2: BC. Identification of potential studies to include and careful revision of the final manuscript: SK, MI, SebK, BC, SE, SO GS, BWR, JGD, HS, VH, MS, SB, JW, MvB. All authors read and approved the final manuscript.

\section{Funding}

Stephan Kruger is supported by the "Else Kröner-Forschungskolleg: Cancer Immunotherapy".

Availability of data and materials

The datasets generated and analysed for Fig. 1 are available in the U.S. National Library of Medicine repository, https://clinicaltrials.gov/

Ethics approval and consent to participate

Not applicable

\section{Consent for publication}

Not applicable

\section{Competing interests}

The authors declare that they have no competing interests.

\section{Author details}

'Department of Medicine III, University Hospital Munich, LMU Munich, Marchioninistr. 15, D-81377 Munich, Germany. ${ }^{2}$ Department of General, Visceral, and Transplantation Surgery, University Hospital, LMU Munich, Munich, Germany. ${ }^{3}$ Center of Integrated Protein Science Munich (CIPS-M) and Division of Clinical Pharmacology, Department of Medicine IV, University Hospital, LMU Munich, Munich, Germany. ${ }^{4}$ Institute of Pathology, LMU Munich, Munich, Germany. ${ }^{5}$ University Hospital of Cologne, Cologne, Germany. ${ }^{6}$ German Cancer Consortium (DKTK), Partner Site Munich; and German Cancer Research Center (DKFZ), Heidelberg, Germany. ${ }^{7}$ Center for Molecular Medicine Cologne (CMMC), Cologne, Germany. ${ }^{8}$ Gene Center LMU, Munich, Germany. 
Received: 31 May 2019 Accepted: 3 June 2019

Published online: 19 June 2019

\section{References}

1. Altmann DM. A Nobel prize-worthy pursuit: cancer immunology and harnessing immunity to tumour neoantigens. Immunology. 2018;155(3):283-4.

2. Gajewski TF, Schreiber H, Fu YX. Innate and adaptive immune cells in the tumor microenvironment. Nat Immunol. 2013;14(10):1014-22.

3. Gong J, Chehrazi-Raffle A, Reddi S, Salgia R. Development of PD-1 and PD-L1 inhibitors as a form of cancer immunotherapy: a comprehensive review of registration trials and future considerations. J Immunother Cancer. 2018;6(1):8.

4. Tang J, Shalabi A, Hubbard-Lucey VM. Comprehensive analysis of the clinical immuno-oncology landscape. Ann Oncol. 2018;29(1):84-91.

5. Apetoh L, Ghiringhelli F, Tesniere A, Obeid M, Ortiz C, Criollo A, et al. Tolllike receptor 4-dependent contribution of the immune system to anticancer chemotherapy and radiotherapy. Nat Med. 2007;13(9):1050-9.

6. Liu WM, Fowler DW, Smith P, Dalgleish AG. Pre-treatment with chemotherapy can enhance the antigenicity and immunogenicity of tumours by promoting adaptive immune responses. Br J Cancer. 2010;102(1):115-23.

7. Hato SV, Khong A, de Vries IJ, Lesterhuis WJ. Molecular pathways: the immunogenic effects of platinum-based chemotherapeutics. Clin Cancer Res. 2014;20(11):2831-7.

8. Galluzzi L, Buque A, Kepp O, Zitvogel L, Kroemer G. Immunological effects of conventional chemotherapy and targeted anticancer agents. Cancer Cell. 2015;28(6):690-714.

9. Tesniere A, Schlemmer F, Boige V, Kepp O, Martins I, Ghiringhelli F, et al. Immunogenic death of colon cancer cells treated with oxaliplatin. Oncogene. 2010;29(4):482-91.

10. Shalapour S, Font-Burgada J, Di Caro G, Zhong Z, Sanchez-Lopez E, Dhar D, et al. Immunosuppressive plasma cells impede T-cell-dependent immunogenic chemotherapy. Nature. 2015;521(7550):94-8.

11. Vincent J, Mignot G, Chalmin F, Ladoire S, Bruchard M, Chevriaux A, et al. 5fluorouracil selectively kills tumor-associated myeloid-derived suppressor cells resulting in enhanced T cell-dependent antitumor immunity. Cancer Res. 2010;70(8):3052-61.

12. Paz-Ares L, Luft A, Vicente D, Tafreshi A, Gumus M, Mazieres J, et al. Pembrolizumab plus chemotherapy for squamous non-small-cell lung Cancer. N Engl J Med. 2018.

13. Gandhi L, Rodríguez-Abreu D, Gadgeel S, Esteban E, Felip E, De Angelis F, et al. Pembrolizumab plus chemotherapy in metastatic non-small-cell lung Cancer. N Engl J Med. 2018;378(22):2078-92.

14. Schmid P, Adams S, Rugo HS, Schneeweiss A, Barrios $\mathrm{CH}$, Iwata $\mathrm{H}$, et al. Atezolizumab and nab-paclitaxel in advanced triple-negative breast Cancer. N Engl J Med. 2018;379(22):2108-21.

15. Burtness B, Harrington KJ, Greil R, Soulières D, Tahara M, De Castro JG, et al. LBA8_PRKEYNOTE-048: phase III study of first-line pembrolizumab (P) for recurrent/metastatic head and neck squamous cell carcinoma (R/M HNSCC). Ann Oncol. 2018;29(suppl_8):mdy424.045-mdy424.045.

16. McCulloch HD. On the analogy between spontaneous recoveries from Cancer and the specific immunity induced by $X$ ray irradiations of the lymphatic glands involved. Br Med J. 1908;2(2494):1146-8

17. Mole RH. Whole body irradiation; radiobiology or medicine? Br J Radiol. 1953:26(305):234-41.

18. Demaria S, Ng B, Devitt ML, Babb JS, Kawashima N, Liebes L, et al. lonizing radiation inhibition of distant untreated tumors (abscopal effect) is immune mediated. Int J Radiat Oncol Biol Phys. 2004;58(3):862-70.

19. Brix N, Tiefenthaller A, Anders H, Belka C, Lauber K. Abscopal, immunological effects of radiotherapy: narrowing the gap between clinical and preclinical experiences. Immunol Rev. 2017;280(1):249-79.

20. Park SS, Dong H, Liu X, Harrington SM, Krco CJ, Grams MP, et al. PD-1 restrains radiotherapy-induced Abscopal effect. Cancer Immunol Res. 2015;3(6):610-9.

21. Hammerich L, Marron TU, Upadhyay R, Svensson-Arvelund J, Dhainaut M, Hussein S, et al. Systemic clinical tumor regressions and potentiation of PD1 blockade with in situ vaccination. Nat Med. 2019:25(5):814-24.

22. McBride SM, Sherman EJ, Tsai CJ, Baxi SS, Aghalar J, Eng J, et al. A phase II randomized trial of nivolumab with stereotactic body radiotherapy (SBRT) versus nivolumab alone in metastatic (M1) head and neck squamous cell carcinoma (HNSCC). J Clin Oncol. 2018;36(15_suppl):6009.

23. Antonia SJ, Villegas A, Daniel D, Vicente D, Murakami S, Hui R, et al. Durvalumab after Chemoradiotherapy in stage III non-small-cell lung Cancer. N Engl J Med. 2017;377(20):1919-29.
24. Antonia SJ, Villegas A, Daniel D, Vicente D, Murakami S, Hui R, et al. Overall survival with Durvalumab after Chemoradiotherapy in stage III NSCLC. N Engl J Med. 2018

25. Faivre-Finn C, Spigel DR, Senan S, Langer CJ, Raben D, Perez B, et al. 13630 Efficacy and safety evaluation based on time from completion of radiotherapy to randomization with durvalumab or placebo in pts from PACIFIC. Ann Oncol. 2018;29(suppl_8):mdy291-mdy.

26. Shrimali RK, Ahmad S, Verma V, Zeng P, Ananth S, Gaur P, et al. Concurrent PD-1 blockade negates the effects of OX40 agonist antibody in combination immunotherapy through inducing T-cell apoptosis. Cancer Immunol Res. 2017;5(9):755-66.

27. Tauriello DVF, Palomo-Ponce S, Stork D, Berenguer-Llergo A, Badia-Ramentol J, Iglesias $\mathrm{M}$, et al. TGFbeta drives immune evasion in genetically reconstituted colon cancer metastasis. Nature. 2018;554(7693):538-43.

28. Beatty GL, Chiorean EG, Fishman MP, Saboury B, Teitelbaum UR, Sun W, et al. CD40 agonists alter tumor stroma and show efficacy against pancreatic carcinoma in mice and humans. Science (New York, NY). 2011;331(6024):1612-6.

29. Luheshi NM, Coates-Ulrichsen J, Harper J, Mullins S, Sulikowski MG, Martin P, et al. Transformation of the tumour microenvironment by a CD40 agonist antibody correlates with improved responses to PD-L1 blockade in a mouse orthotopic pancreatic tumour model. Oncotarget. 2016;7(14):18508-20.

30. O'Hara MH. CT004 - a phase Ib study of CD40 agonistic monoclonal antibody APX005M together with gemcitabine (gem) and nab-paclitaxel (NP) with or without nivolumab (Nivo) in untreated metastatic ductal pancreatic adenocarcinoma (PDAC) patients. In: Annual Meeting of the American Association for Cancer Research; Atlanta; 2019.

31. Huang AC, Postow MA, Orlowski RJ, Mick R, Bengsch B, Manne S, et al. T-cell invigoration to tumour burden ratio associated with anti-PD-1 response. Nature. 2017;545(7652):60-5.

32. Joseph RW, Elassaiss-Schaap J, Kefford R, Hwu WJ, Wolchok JD, Joshua AM, et al. Baseline tumor size is an independent prognostic factor for overall survival in patients with melanoma treated with Pembrolizumab. Clin Cancer Res. 2018;24(20):4960-7.

33. Garcia CA, El-Ali A, Rath TJ, Contis LC, Gorantla V, Drappatz J, et al. Neurologic immune-related adverse events associated with adjuvant ipilimumab: report of two cases. J Immunother Cancer. 2018;6(1):83.

34. Eggermont AM, Chiarion-Sileni V, Grob JJ, Dummer R, Wolchok JD, Schmidt $\mathrm{H}$, et al. Adjuvant ipilimumab versus placebo after complete resection of high-risk stage III melanoma (EORTC 18071): a randomised, double-blind, phase 3 trial. Lancet Oncol. 2015;16(5):522-30.

35. Eggermont AM, Chiarion-Sileni V, Grob JJ, Dummer R, Wolchok JD, Schmidt $\mathrm{H}$, et al. Prolonged survival in stage III melanoma with Ipilimumab adjuvant therapy. N Engl J Med. 2016;375(19):1845-55.

36. Weber J, Mandala M, Del Vecchio M, Gogas HJ, Arance AM, Cowey CL, et al. Adjuvant Nivolumab versus Ipilimumab in resected stage III or IV melanoma. N Engl J Med. 2017;377(19):1824-35.

37. Liu J, Blake SJ, Yong MC, Harjunpaa H, Ngiow SF, Takeda K, et al. Improved efficacy of neoadjuvant compared to adjuvant immunotherapy to eradicate metastatic disease. Cancer Discovery. 2016;6(12):1382-99.

38. Blank CU, Rozeman EA, Fanchi LF, Sikorska K, van de Wiel B, Kvistborg P, et al. Neoadjuvant versus adjuvant ipilimumab plus nivolumab in macroscopic stage III melanoma. Nat Med. 2018.

39. Forde PM, Chaft JE, Smith KN, Anagnostou V, Cottrell TR, Hellmann MD, et al. Neoadjuvant PD-1 blockade in Resectable lung Cancer. N Engl J Med. 2018;378(21):1976-86.

40. Bell RB, Duhen R, Leidner RS, Curti BD, Ballesteros-Merino C, Piening B, et al. Neoadjuvant anti-OX40 (MEDI6469) prior to surgery in head and neck squamous cell carcinoma. J Clin Oncol. 2018;36(15_suppl):6011.

41. Chalabi M, Fanchi LF, Van den Berg JG, Beets GL, Lopez-Yurda M, Aalbers AG, et al. LBA37_PRNeoadjuvant ipilimumab plus nivolumab in early stage colon cancer. Ann Oncol. 2018;29(suppl_8):mdy424.047-mdy424.047.

42. Garcia-Murillas I, Schiavon G, Weigelt B, Ng C, Hrebien S, Cutts RJ, et al. Mutation tracking in circulating tumor DNA predicts relapse in early breast cancer. Sci Transl Med. 2015;7(302):302ra133.

43. Alexandrov LB, Nik-Zainal S, Wedge DC, Aparicio SAJR, Behjati S, Biankin AV, et al. Signatures of mutational processes in human cancer. Nature. 2013;500:415.

44. Kadia TM, Cortes JE, Ghorab A, Ravandi F, Jabbour E, Daver NG, et al. Nivolumab (Nivo) maintenance (maint) in high-risk (HR) acute myeloid leukemia (AML) patients. J Clin Oncol. 2018;36(15_suppl):7014.

45. Colwell J. IS PD-L1 expression a biomarker of response? Cancer discovery. $2015 ; 5(12): 1232$ 
46. Fabrizio DA, George TJ Jr, Dunne RF, Frampton G, Sun J, Gowen K, et al. Beyond microsatellite testing: assessment of tumor mutational burden identifies subsets of colorectal cancer who may respond to immune checkpoint inhibition. J Gastrointest Oncol. 2018;9(4):610-7.

47. Endris V, Buchhalter I, Allgauer M, Rempel E, Lier A, Volckmar AL, et al. Measurement of tumor mutational burden (TMB) in routine molecular diagnostics: in silico and real-life analysis of three larger gene panels. Int J Cancer. 2019;144(9):2303-12.

48. Rizvi NA, Hellmann MD, Snyder A, Kvistborg P, Makarov V, Havel JJ, et al. Cancer immunologyMutational landscape determines sensitivity to PD-1 blockade in non-small cell lung cancer. Science (New York, NY). 2015;348(6230):124-8.

49. Chan TA, Yarchoan M, Jaffee E, Swanton C, Quezada SA, Stenzinger A. Development of tumor mutation burden as an immunotherapy biomarker: utility for the oncology clinic. Ann Oncol. 2019;30(1):44-56.

50. Salem ME, Puccini A, Grothey A, Xiu J, Goldberg R. Kim ES, et al 1835PDComparative molecular analysis between microsatellite instabilityhigh (MSI-H) tumors with high tumor mutational burden (TMB-H) versus MSI-H tumors with TMB-intermediate/low. Ann Oncol. 2018;29(suppl_8): mdy303.005-mdy303.005.

51. Carbone DP, Reck M, Paz-Ares L, Creelan B, Horn L, Steins M, et al. First-line Nivolumab in stage IV or recurrent non-small-cell lung Cancer. N Engl J Med. 2017;376(25):2415-26

52. Hellmann MD, Ciuleanu T-E, Pluzanski A, Lee JS, Otterson GA, AudigierValette $C$, et al. Nivolumab plus Ipilimumab in lung Cancer with a high tumor mutational burden. N Engl J Med. 2018;378(22):2093-104.

53. McDermott DF, Huseni MA, Atkins MB, Motzer RJ, Rini BI, Escudier B, et al. Clinical activity and molecular correlates of response to atezolizumab alone or in combination with bevacizumab versus sunitinib in renal cell carcinoma. Nat Med. 2018;24(6):749-57.

54. Yang W, Lee KW, Srivastava RM, Kuo F, Krishna C, Chowell D, et al. Immunogenic neoantigens derived from gene fusions stimulate T cell responses. Nature medicine. 2019.

55. Frigola X, Inman BA, Lohse CM, Krco C, Cheville JC, Thompson RH, et al. Identification of a soluble form of B7-H1 that retains immunosuppressive activity and is associated with aggressive renal cell carcinoma. Clin Cancer Res. 2011;17(7):1915-23.

56. Kruger S, Legenstein ML, Rosgen V, Haas M, Modest DP, Westphalen CB, et al. Serum levels of soluble programmed death protein 1 (SPD-1) and soluble programmed death ligand 1 (SPD-L1) in advanced pancreatic cancer. Oncoimmunology. 2017;6(5):e1310358.

57. Costantini A, Julie C, Dumenil C, Helias-Rodzewicz Z, Tisserand J, Dumoulin J, et al. Predictive role of plasmatic biomarkers in advanced non-small cell lung cancer treated by nivolumab. Oncoimmunology. 2018;7(8):e1452581.

58. Gandara DR, Paul SM, Kowanetz M, Schleifman E, Zou W, Li Y, et al. Blood-based tumor mutational burden as a predictor of clinical benefit in non-small-cell lung cancer patients treated with atezolizumab. Nat Med. 2018;24(9):1441-8.

59. Kim ES, Velcheti V, Mekhail T, Leal TA, Dowell JE, Tsai ML, et al. LBA55Primary efficacy results from B-F1RST, a prospective phase II trial evaluating blood-based tumour mutational burden (bTMB) as a predictive biomarker for atezolizumab (atezo) in $1 \mathrm{~L}$ non-small cell lung cancer (NSCLC). Ann Oncol. 2018;29(suppl_8):mdy424.067-mdy424.067.

60. Kruger S, Heinemann V, Ross C, Diehl F, Nagel D, Ormanns S, et al. Repeated mutKRAS ctDNA measurements represent a novel and promising tool for early response prediction and therapy monitoring in advanced pancreatic cancer. Ann Oncol. 2018;29(12):2348-55.

61. Lee JH, Long GV, Boyd S, Lo S, Menzies AM, Tembe V, et al. Circulating tumour DNA predicts response to anti-PD1 antibodies in metastatic melanoma. Ann Oncol. 2017:28(5):1130-6.

62. Goldberg SB, Narayan A, Kole AJ, Decker RH, Teysir J, Carriero NJ, et al. Early assessment of lung Cancer immunotherapy response via circulating tumor DNA. Clin Cancer Res. 2018:24(8):1872-80.

63. Guibert N, Mazieres J, Delaunay M, Casanova A, Farella M, Keller L, et al. Monitoring of KRAS-mutated ctDNA to discriminate pseudo-progression from true progression during anti-PD-1 treatment of lung adenocarcinoma. Oncotarget. 2017:8(23):38056-60.

64. Ricciuti B, Giglio AD, Brambilla M, Bassanelli M, Metro G, Ludovini V, et al. Immune-related adverse events to predict survival in patients with advanced non-small cell lung cancer treated with nivolumab: a multicenter analysis. J Clin Oncol. 2018;36(15_suppl):9084.

65. Haratani K, Hayashi H, Chiba Y, et al. Association of immune-related adverse events with nivolumab efficacy in non-small-cell lung cancer. JAMA Oncol. 2018:4(3):374-8.
66. Kfoury M, Voisin AL, Najean M, Champiat S, Laghouati S, Michot JM, et al. 1141PDAssociation between immune-related adverse events and efficacy in patients treated with anti-PD-(L)1. Ann Oncol. 2018;29(suppl_8):mdy288.014mdy288.014.

67. Arbour KC, Mezquita L, Long N, Rizvi H, Auclin E, Ni A, et al. Deleterious effect of baseline steroids on efficacy of PD-(L)1 blockade in patients with NSCLC. J Clin Oncol. 2018;36(15_suppl):9003.

68. Verzoni E, Carteni G, Cortesi E, Giannarelli D, De Giglio A, Sabbatini R, et al. Real-world efficacy and safety of nivolumab in previously-treated metastatic renal cell carcinoma, and association between immune-related adverse events and survival: the Italian expanded access program. J Immunother Cancer. 2019;7(1):99

69. Haanen JBAG, Carbonnel F, Robert C, Kerr KM, Peters S, Larkin J, et al. Management of toxicities from immunotherapy: ESMO clinical practice guidelines for diagnosis, treatment and follow-upt. Ann Oncol. 2017; 28(suppl_4):iv119-iv42.

70. Danlos F-X, Voisin A-L, Dyevre V, Michot J-M, Routier E, Taillade L, et al. Safety and efficacy of anti-programmed death 1 antibodies in patients with cancer and pre-existing autoimmune or inflammatory disease. Eur J Cancer. 2018;91:21-9.

71. Gobert A, Veyri M, Lavolé A, Montaudié H, Cloarec N, Doucet L, et al. 1213P_PRTolerance and efficacy of immune-checkpoint inhibitors for cancer in people living with HIV (PWHIV). Ann Oncol. 2018;29(suppl_8):mdy424.051mdy424.051.

72. Maude SL, Laetsch TW, Buechner J, Rives S, Boyer M, Bittencourt H, et al. Tisagenlecleucel in children and young adults with B-cell lymphoblastic leukemia. N Engl J Med. 2018;378(5):439-48.

73. Schuster SJ, Bishop MR, Tam CS, Waller EK, Borchmann P, McGuirk JP, et al. Tisagenlecleucel in adult relapsed or refractory diffuse large B-cell lymphoma. N Engl J Med. 2019;380(1):45-56.

74. Neelapu SS, Locke FL, Bartlett NL, Lekakis LJ, Miklos DB, Jacobson CA, et al. Axicabtagene Ciloleucel CAR T-cell therapy in refractory large B-cell lymphoma. N Engl J Med. 2017;377(26):2531-44.

75. Results retrieved from ClinicalTrials.gov on 2019-05-03, search: "other terms" = "car t cells" [internet]. 2019 [cited 2019-05-03].

76. Hartmann J, Schussler-Lenz M, Bondanza A, Buchholz CJ. Clinical development of CAR T cells-challenges and opportunities in translating innovative treatment concepts. EMBO Mol Med. 2017:9(9):1183-97.

77. Grupp SA, Maude SL, Rives S, Baruchel A, Boyer MW, Bittencourt H, et al. Updated analysis of the efficacy and safety of Tisagenlecleucel in pediatric and young adult patients with relapsed/refractory $(r / r)$ acute lymphoblastic leukemia. Blood. 2018;132(Suppl 1):895.

78. Borchmann P, Tam CS, Jäger U, McGuirk JP, Holte H, Waller EK, et al. An updated analysis of JULIET, a global pivotal phase 2 trial of Tisagenlecleucel in adult patients with relapsed or refractory diffuse large B-cell lymphoma. Abstract S799, presented at the annual congress of the European Hematology Association (EHA) 2018.

79. Schuster SJ, Bishop MR, Tam C, Borchmann P, Jaeger U, Waller EK, et al. Sustained disease control for adult patients with relapsed or refractory diffuse large B-cell lymphoma: an updated analysis of Juliet, a global pivotal phase 2 trial of Tisagenlecleucel. Blood. 2018;132(Suppl 1):1684.

80. Neelapu SS, Ghobadi A, Jacobson CA, Miklos DB, Lekakis LJ, Oluwole OO, et al. 2-year follow-up and high-risk subset analysis of Zuma-1, the pivotal study of Axicabtagene Ciloleucel (Axi-Cel) in patients with refractory large B cell lymphoma. Blood. 2018;132(Suppl 1):2967.

81. Nastoupil LJ, Jain MD, Spiegel JY, Ghobadi A, Lin Y, Dahiya S, et al. Axicabtagene Ciloleucel (Axi-cel) CD19 chimeric antigen receptor (CAR) Tcell therapy for relapsed/refractory large B-cell lymphoma: real world experience. Blood. 2018;132(Suppl 1):91.

82. Summers C, Annesley C, Bleakley M, Dahlberg A, Jensen MC, Gardner R. Long term follow-up after SCRI-CAR19v1 reveals late recurrences as well as a survival advantage to consolidation with HCT after CAR T cell induced remission. Blood. 2018;132(Suppl 1):967

83. Sano D, Nastoupil L, Fowler NH, Fayad L, Hagemeister FB, Lee HJ, et al. Safety of Axicabtagene Ciloleucel CD19 CAR T-cell therapy in elderly patients with relapsed or refractory large B-cell lymphoma. Blood. 2018; 132(Suppl 1):96

84. Faramand R, Kotani H, Morrissey D, Yu B, Locke FL, Jain MD, et al. Prediction of CAR T-related toxicities in R/R DLBCL patients treated with Axicabtagene Ciloleucel using point of care cytokine measurements. Blood. 2018; 132(Suppl 1):95. 
85. Ying $Z$, Huang $X F$, Xiang $X$, Liu $Y$, Kang $X$, Song $Y$, et al. A safe and potent anti-CD19 CAR T cell therapy. Nature medicine. 2019.

86. Du H, Hirabayashi K, Ahn S, Kren NP, Montgomery SA, Wang X, et al. Antitumor responses in the absence of toxicity in solid tumors by targeting $\mathrm{B} 7-\mathrm{H} 3$ via chimeric antigen receptor T cells. Cancer Cell. 2019;35(2):221-37 e8.

87. Gargett T, Brown MP. The inducible caspase-9 suicide gene system as a "safety switch" to limit on-target, off-tumor toxicities of chimeric antigen receptor T cells. Front Pharmacol. 2014;5:235.

88. Mestermann K, Julian R, Silke F, Hermann E, Hudecek M. Abstract A037: A novel pharmacologic "ON/OFF" switch to modulate CAR-T-cell function in vitro and in vivo. Cancer Immunol Res. 2019;7(2 Supplement):A037-A.

89. Geyer MB, Riviere I, Senechal B, Wang X, Wang Y, Purdon TJ, et al. Safety and tolerability of conditioning chemotherapy followed by CD19-targeted CAR T cells for relapsed/refractory CLL. JCI Insight. 2019;5.

90. Kim MY, Yu KR, Kenderian SS, Ruella M, Chen S, Shin TH, et al. Genetic inactivation of CD33 in hematopoietic stem cells to enable CAR T cell immunotherapy for acute myeloid leukemia. Cell. 2018;173(6):1439-53 e19.

91. Rafiq S, Yeku OO, Jackson HJ, Purdon TJ, van Leeuwen DG, Drakes DJ, et al. Targeted delivery of a PD-1-blocking scFv by CAR-T cells enhances antitumor efficacy in vivo. Nat Biotechnol. 2018;36(9):847-56.

92. Chmielewski M, Abken H. CAR T cells releasing IL-18 convert to T-bet(high) FoxO1(low) effectors that exhibit augmented activity against advanced solid tumors. Cell Rep. 2017;21(11):3205-19.

93. Chmielewski M, Hombach AA, Abken H. Of CARs and TRUCKs: chimeric antigen receptor (CAR) T cells engineered with an inducible cytokine to modulate the tumor stroma. Immunol Rev. 2014;257(1):83-90.

94. Kloess S, Kretschmer A, Stahl L, Fricke S, Koehl U. CAR-expressing natural killer cells for Cancer retargeting. Transfus Med Hemother. 2019:46(1):4-13.

95. Akce M, Zaidi MY, Waller EK, El-Rayes BF, Lesinski GB. The potential of CAR T cell therapy in pancreatic Cancer. Front Immunol. 2018;9:2166.

96. Tokarew N, Ogonek J, Endres S, von Bergwelt-Baildon M, Kobold S. Teaching an old dog new tricks: next-generation CAR T cells. Br J Cancer. 2019;120(1):26-37.

97. Legut M, Dolton G, Mian AA, Ottmann OG, Sewell AK. CRISPR-mediated TCR replacement generates superior anticancer transgenic T cells. Blood. 2018; 131(3):311-22

98. Raman MC, Rizkallah PJ, Simmons R, Donnellan Z, Dukes J, Bossi G, et al. Direct molecular mimicry enables off-target cardiovascular toxicity by an enhanced affinity TCR designed for cancer immunotherapy. Sci Rep. 2016;6:18851.

99. Rosenberg SA, Restifo NP. Adoptive cell transfer as personalized immunotherapy for human cancer. Science (New York, NY). 2015;348(6230):62-8.

100. Leung W, Heslop HE. Adoptive immunotherapy with antigen-specific T cells expressing a native TCR. Cancer Immunol Res. 2019;7(4):528-33.

101. Dahl K, Karlsson M, Marits P, Hoffstedt A, Winqvist O, Thörn M. Metinel node- - the first lymph node draining a metastasis-contains tumor-reactive lymphocytes. Ann Surg Oncol. 2008;15(5):1454-63.

102. Karlsson M, Marits P, Dahl K, Dagöö T, Enerbäck S, Thörn M, et al. Pilot study of sentinel-node-based adoptive immunotherapy in advanced colorectal cancer. Ann Surg Oncol. 2010;17(7):1747-57.

103. Marits $P$, Karlsson M, Dahl K, Larsson P, Wanders A, Thorn M, et al. Sentinel node lymphocytes: tumour reactive lymphocytes identified intraoperatively for the use in immunotherapy of colon cancer. Br J Cancer. 2006;94(10):1478-84.

104. Tran E, Turcotte S, Gros A, Robbins PF, Lu YC, Dudley ME, et al. Cancer immunotherapy based on mutation-specific CD4+ T cells in a patient with epithelial cancer. Science (New York, NY). 2014;344(6184):641-5.

105. Tran E, Robbins PF, Lu YC, Prickett TD, Gartner JJ, Jia L, et al. T-cell transfer therapy targeting mutant KRAS in Cancer. N Engl J Med. 2016;375(23):2255-62.

106. Zacharakis N, Chinnasamy H, Black M, Xu H, Lu YC, Zheng Z, et al. Immune recognition of somatic mutations leading to complete durable regression in metastatic breast cancer. Nat Med. 2018:24(6):724-30

107. Sarnaik AA TS, Davar D, editor Safety and efficacy of cryopreserved autologous tumor infiltrating lymphocyte therapy (LN-144, lifileucel) in advanced metastatic melanoma patients following progression on checkpoint inhibitors. 33rd annual meeting \& pre-conference programs of the Society for Immunotherapy of Cancer (SITC 2018); 2018 November 06.

Ready to submit your research? Choose BMC and benefit from:

- fast, convenient online submission

- thorough peer review by experienced researchers in your field

- rapid publication on acceptance

- support for research data, including large and complex data types

- gold Open Access which fosters wider collaboration and increased citations

- maximum visibility for your research: over $100 \mathrm{M}$ website views per year

At BMC, research is always in progress.

Learn more biomedcentral.com/submissions 